\title{
Síndrome Demencial como Fator de Risco para Penfigoide Bolhoso
}

\section{Dementia as a Risk Factor for Bullous Pemphigoid}

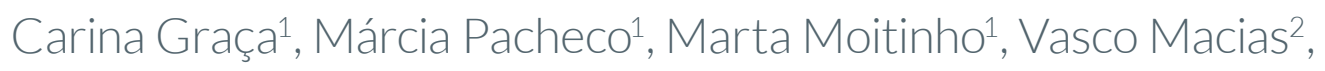
Sunita Dessai², José Barata ${ }^{1}$

Autor Correspondente: Carina Graça [carinagouveia@hotmail.com; carina.graca@hvfx.pt] Rua Alves Redol, lote 123, $7^{\circ}$ direito, 2600-100 Vila Franca de Xira, Portugal

\section{RESUMO}

O penfigoide bolhoso é uma patologia cutânea autoimune adquirida, mais frequente nos idosos. Estudos recentes demonstraram a associação causal entre a ocorrência de penfigoide bolhoso em doentes com síndromes demenciais. Apresentamos o caso de uma doente do sexo feminino, 80 anos, com antecedentes de doença de Parkinson, internada por dermatose bolhosa extensa, constituída por placas urticariformes e bolhas tensas com halo eritematoso, sem envolvimento das mucosas. Após confirmação por biópsia cutânea, imunofluorescência direta positiva e exclusão de outras causas, foi feito o diagnóstico de penfigoide bolhoso. Foi instituída terapêutica com prednisolona, azatriopina e doxiciclina, verificando-se evolução clínica favorável. Consideramos o presente caso clínico pertinente, pois realça a importância do diagnóstico precoce de penfigoide bolhoso. O atraso no início do tratamento pode levar a complicações graves, com elevada morbi-mortalidade.

PALAVRAS-CHAVE: Demência; Doença de Parkinson; Fatores de Risco; Idoso; Penfigoide Bolhoso

\section{ABSTRACT}

Bullous pemphigoid is an acquired autoimmune skin disease, more often in the elderly. Recent studies have shown the association between bullous pemphigoid and neurological diseases. We present the case of an 80-year-old female, with prior history of Parkinson's disease, admitted in our ward with an extensive bullous dermatosis involving the trunk, upper limbs and thighs, consisting of erythematous vesicular lesions, with no involvement of the mucosae. After the confirmation by the skin biopsy, positive direct immunofluorescence and exclusion of other causes, the diagnosis of bullous pemphigoid was established. She was submitted to targeted treatment with prednisolone, azathioprine and doxycycline, with favorable clinical evolution. This clinical case highlights the importance of early diagnosis in patients with demential diseases. The delay in treatment of this autoimmune skin can lead to serious complications.

KEYWORDS: Aged; Dementia; Parkinson Disease; Pemphigoid, Bullous; Risk Factors 


\section{INTRODUÇÃO}

O penfigoide bolhoso (PB) é uma patologia cutânea autoimune adquirida, caraterizado pela presença de bolhas cutâneas extensas generalizadas, frequente na população idosa. ${ }^{1,2}$ Vários estudos recentes demonstraram a associação entre a ocorrência de PB em doentes com síndromes neurológicas como esclerose múltipla, doença de Parkinson, epilepsia e doenças cerebrovasculares..$^{1-5}$ Os mecanismos fisiopatológicos subjacentes a este processo ainda não estão totalmente esclarecidos. A hipótese mais plausível tem como base a reatividade imunológica cruzada entre autoanticorpos das células cutâneas e do sistema nervoso central, potenciada pela perda de tolerância imunológica que está associada ao processo de envelhecimento e ao aparecimento de síndromes demenciais. ${ }^{1,3}$

\section{CASO CLÍNICO}

Doente do sexo feminino, 80 anos, raça negra, totalmente dependente de terceiros nas atividades de vida diária. Antecedentes patológicos conhecidos de doença de Parkinson em estádio 5 (escala modificada de Hoehn e Yahr), medicada cronicamente com levodopa/carbidopa (Sinemet ${ }^{\circledR}$ ) 100/25 mg, duas vezes por dia. Apresentava um quadro de dermatose bolhosa extensa, com cerca de duas semanas de evolução, envolvendo o tronco, membros superiores e coxas, constituída por bolhas tensas com halo eritematoso e de fundo sangrante, distribuídas pela região anterior do tronco, membros superiores e coxas (Fig. 1). Observavam-se ainda múltiplas placas eritematosas urticariformes, de superfície lisa, dispersas sobretudo na região dorsal. Sem envolvimento das mucosas oral e genital. Na anamnese efetuada, foi negada história de febre ou de outra sintomatologia associada. Não foram detetados possíveis fatores precipitantes, nomeadamente história sugestiva de neoplasia, exposição a tóxicos ou introdução recente de medicação. O restante exame objetivo não revelou alterações. A doente foi inicialmente observada em consulta externa de Dermatologia, optando-se posteriormente pelo seu internamento no serviço de Medicina Interna para estudo etiológico, otimização terapêutica e cuidados de penso, tendo em conta a extensão da dermatose. Do estudo complementar de diagnóstico efetuado, destaca-se biópsia cutânea compatível com penfigoide bolhoso (imunofluorescência direta positiva, com deposição linear de C3 ao longo da junção dermoepidérmica). Os anticorpos anti-desmogelina e anti-BP 180 foram negativos. A restante avaliação laboratorial não revelou alterações relevantes.

Atendendo ao estado geral da doente e à ausência de outros sintomas associados, admitiu-se diagnóstico de

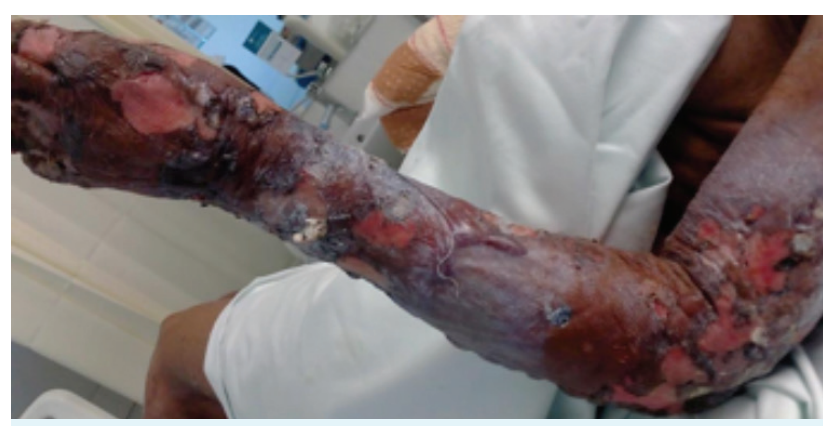

FIGURA 1. Dermatose bolhosa, membro superior esquerdo.

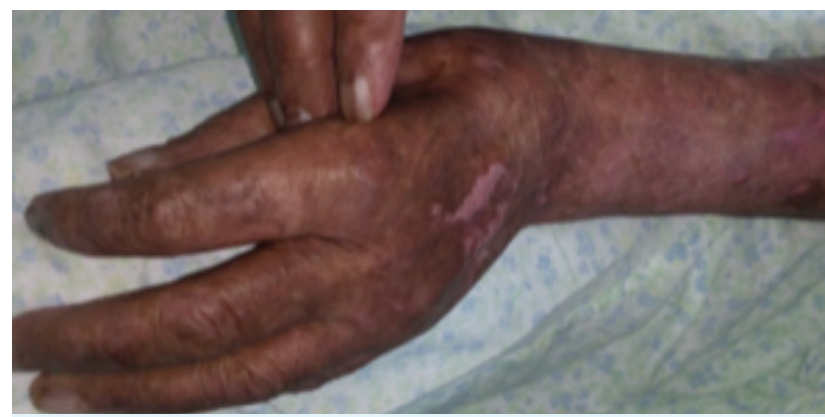

FIGURA 2. Cicatrização completa das lesões do membro superior esquerdo.

PB no contexto de síndrome demencial em estádio avançado. Iniciou terapêutica com prednisolona $(0,5 \mathrm{mg} / \mathrm{kg}$, escalada até $1 \mathrm{mg} / \mathrm{kg}$ ) e cuidados cutâneos locais, nomeadamente aspiração de novas bolhas e aplicação tópica de permanganato e óxido de zinco, sem resposta clínica após duas semanas. Atendendo à refratariedade à corticoterapia, associou-se azatioprina (até um máximo de 150 mg/dia) e doxiciclina 100 mg/dia, verificando-se então evolução clínica favorável, sem aparecimento de novas bolhas e com reepitelização completa das lesões existentes, no período de duas semanas (Fig. 2). Teve alta clínica no $47^{\circ}$ dia de internamento, mantendo terapêutica com azatioprina 150 mg/dia e esquema de desmame de corticoterapia, sem necessidade de cuidados de penso, mantendo apenas necessidade de aplicação tópica de emoliente. Foi referenciada para a consulta de Dermatologia para vigilância e continuação de cuidados.

\section{DISCUSSÃO}

O penfigoide bolhoso constitui uma dermatose bolhosa autoimune que habitualmente se manifesta nas faixas etárias superiores (60-80 anos). ${ }^{6}$ A incidência de PB está estimada em dois a 14 novos casos por milhão, sem predominância por género. ${ }^{7}$ Manifesta-se através de uma erupção cutânea polimórfica, tipicamente constituída por bolhas subepidérmicas tensas, que podem ter distribuição localizada ou generalizada. O envolvimento das mucosas é pouco comum, estando descrito em cerca de 10 a 25\% dos casos. ${ }^{6,8,9} \mathrm{O}$ diagnóstico é determinado 
histologicamente, através da biópsia cutânea e da imunofluorescência direta.6,9

Segundo a literatura, existem certos fatores de risco que contribuem para o aparecimento de PB, incluindo consumo crónico de fármacos (por ex.: aspirina, captopril, furosemida, penicilina) e a presença de comorbilidades como síndromes linfoproliferativas, diabetes mellitus e doenças neurodegenerativas. ${ }^{5,7}$ A relação causal entre as doenças do foro neurológico e a ocorrência de penfigoide bolhoso tem sido muito investigada nos últimos anos. ${ }^{1-5,7}$ Embora a sua patogénese ainda não esteja totalmente esclarecida, acredita-se que os processos neurodegenerativos possam predispor o desenvolvimento de PB, através da ativação de mecanismos autoimunes, que produzem autoanticorpos contra antigénios presentes nas células cutâneas e do sistema nervoso central. ${ }^{1-4}$ Estas observações são reforçadas pelo facto de ambos os tecidos (cutâneo e sistema nervoso) serem originados a partir da crista neural. Além disso, acredita-se que todo este processo é potenciado pela perda de tolerância imunológica fisiológica que está associada ao processo de envelhecimento. ${ }^{1,3}$

O tratamento de primeira linha, recomendado pela literatura, consiste na corticoterapia sistémica oral (prednisolona 0,5 a $1 \mathrm{mg} / \mathrm{kg}$ ). A associação com outros agentes imunossupressores (azatioprina, micofenolato mofetil) e com antimicrobianos (tetraciclinas), deverá ser efetuada se se verificar refratariedade à monoterapia com corticoides.

Consideramos o presente caso clínico pertinente dada a prevalência crescente de síndromes demenciais, reflexo do rápido envelhecimento da população. Salienta-se a importância da abordagem multidisciplinar efetuada (Medicina Interna, Dermatologia, equipa de enfermagem), que levaram a um correto diagnóstico e à instituição de terapêutica precoce, atendendo à elevada taxa de complicações graves com potencial de disfunção multiorgânica.

CONFLITOS DE INTERESSE: Os autores declaram não ter qualquer conflito de interesse na realização do presente trabalho.

FONTES DE FINANCIAMENTO: Não houve qualquer fonte de financiamento na realização do presente trabalho.

CONFIDENCIALIDADE DOS DADOS: Os autores declaram ter seguido os protocolos da sua instituição acerca da publicação dos dados de doentes.
PROTEÇÃO DE PESSOAS E ANIMAIS: Os autores declaram que os procedimentos seguidos na elaboração do presente trabalho estão em conformidade com as normas das comissões de investigação clínica e de ética, bem como da declaração de Helsínquia e da AssociaçãoMédica Mundial.

CONFLICTS OF INTEREST: The authors declare that they have no conflicts of interest.

FINANCIAL SUPPORT: This work has not received any contribution, grant or scholarship.

CONFIDENTIALITY OF DATA: The authors declare that they have followed the protocols of their work center on the publication of data from patients.

PROTECTION OF HUMAN AND ANIMAL SUBJECTS: The authors declare that the procedures followed were in accordance with the regulations of the relevant clinical research ethics committee and with those of the Code of Ethics of the World Medical Association (Declaration of Helsinki).

\section{REFERÊNCIAS}

1. Stinco G, Coddutt R, Scarbolo M, Valent F, Patrone P. A Retrospective epidemiological study on the association of bullous pemphigoid and neurological diseases. Acta Derm Venereol. 2005;85:136-9.

2. Taghipour K, Chi CC, Vincent A, Groves RW, Venning V, Wojnarowska $F$. The association of bullous pemphigoid with cerebrovascular disease and dementia. Arch Dermatol. 2010;146:1251-4.

3. Sinead M, Langan, Richard W, Groves, West J. The relationship between neurological disease and bullous pemphigoid: a population-based case-control study. J Invest Dermatol. 2011;131:631-6.

4. Cordel N. Neurological disorders in patients with bullous pemphigoid. Dermatology. 2007;215:187-91.

5. Bastuji-Garin S, Joly P, Lemordant P, Sparsa A, Bedane C, Delaporte E, et al. Risk factors for bullous pemphigoid in the elderly: a prospective case-control study. J Invest Dermatol. 2011;131:637-43.

6. Stanley JR. Bullous pemphigoid. In: Freedberg IM, Eisen AZ, Wolff $\mathrm{K}$, et al, editors. Fitzpatrick's Dermatology in general medicine. 5th ed. New York: McGraw-Hill; 1999. p.666-79.

7. Teixeira V, Cabral R, Brites MM, Vieira R, Figueiredo A. Bullous pemphigoid and comorbidities: a case-control study in Portuguese patients. An Bras Dermatol. 2014;89:274-8.

8. Cunha PR, Barraviera SR. Dermatoses bolhosas auto-imunes. An Bras Dermatol. 2009;84:111-24.

9. Ruocco E, Wolf R, Caccavale S, Brancaccio G, Ruocco V, Lo Schiavo A. Bullous pemphigoid: associations and management guidelines: facts and controversies. Clin Dermatol. 2013;31:400-12. 